\title{
Dialisi e qualità di vita: una indagine presso il Centro Dialisi di Rimini
}

\author{
Marina Sgreccia ${ }^{1}$, Laura Capicchioni ${ }^{2}$, Ester Chierighini ${ }^{3}$, Roberto Boccadoro ${ }^{4}$ \\ ${ }^{1}$ Coordinatore Infermieristico Dialisi, U.O. Nefrologia e Dialisi, Ospedale degli Infermi - Rimini, Azienda USL della Romagna, Rimini \\ ${ }^{2}$ Studentessa Corso di Laurea Infermieristica Università di Bologna Campus di Rimini, Rimini \\ ${ }^{3}$ Infermiera Referente, Ambulatorio Dialisi Peritoneale U.O. Nefrologia e Dialisi, Ospedale degli Infermi - Rimini, Azienda USL della Romagna, Rimini \\ ${ }^{4}$ Medico Nefrologo U.O. Nefrologia e Dialisi, Ospedale degli Infermi - Rimini, Azienda USL della Romagna, Rimini
}

\begin{abstract}
Dialysis and quality of life: a survey at the Rimini dialysis center
The concept of quality of life includes several aspects of the physical and psychological status. In a medical setting the evaluation of health-related quality of life comprises physiopathological and clinical aspects, as well as patient well-being and functioning. The aims of this study were to carry out an updated evaluation of the health status and quality of life of chronic hemodialysis patients and compare these with peritoneal dialysis patients at the ASL Rimini Dialysis Department. The final purpose is to obtain data comparable with other studies or other populations, and to identify possible actions or interventions.
\end{abstract}

Keywords: Hemodialysis, Peritoneal dialysis, Health-related quality of life

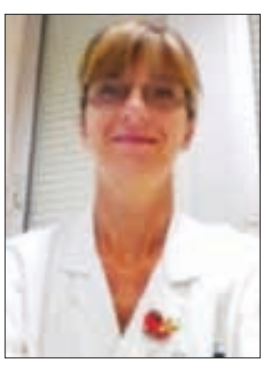

Marina Sgreccia

\section{Introduzione}

Nel 2009 l'incidenza cumulativa di nuovi pazienti in trattamento dialitico in Italia, stimata sulla popolazione disponibile al Registro Italiano Dialisi e Trapianto e corretta per la copertura percentuale dei singoli Registri regionali, era pari a 160 pazienti per milione di popolazione (pmp). Nel nostro Paese i pazienti trattati con la dialisi peritoneale rappresentano il $10 \%$ circa di tutta la popolazione dializzata (1).

Secondo quanto emerge dal Registro Regionale dell'Emilia Romagna, il 31 dicembre 2010 l'Emilia Romagna ha una popolazione generale di 4.432 .439 abitanti e ha oltre 3000 pazienti in trattamento dialitico cronico: 2891 pazienti eseguono l'emodialisi e 290 sono in dialisi peritoneale. Oltre 1200 pazienti sono invece portatori di un trapianto renale.

I pazienti in dialisi extracorporea eseguono i trattamenti dialitici presso i Centri Dialisi ospedalieri o ad assistenza

\footnotetext{
Accepted: January 15, 2015

Published online: February 26, 2015

Indirizzo per la corrispondenza:

Marina Sgreccia

Centro Dialisi Rimini

Ospedale degli Infermi

Via Settembrini, 2

47900 Rimini

marina.sgreccia@auslromagna.it
}

limitata (CAL) o ad assistenza decentrata (CAD) delle 13 Unità Operative complesse della regione. La dialisi peritoneale, che costituisce il 9,11\% dei trattamenti dialitici, viene eseguita al domicilio del paziente o in via manuale (CAPD) in un quarto dei pazienti o in via automatizzata (APD) nei restanti tre quarti. La percentuale di dialisi peritoneale risulta leggermente inferiore al dato nazionale del 10,5\% riportato dal RIDT (Registro Italiano Dialisi Trapianto). Non tutte le U.O. di Nefrologia hanno la stessa "vocazione" per la dialisi peritoneale; in alcune la dialisi peritoneale supera anche il $20 \%$ dei trattamenti dialitici, mentre in altre è a livelli del tutto trascurabili (2).

Con il recente aumento della prevalenza delle malattie croniche, il risultato delle cure non va più valutato "dal punto di vista del medico", e cioè solo sulla base della normalizzazione degli esami e dei sintomi o del prolungamento della sopravvivenza, ma anche "dal punto di vista del paziente", cioè tenendo presente l'effetto che la malattia e le cure hanno sul paziente, inteso come individuo inserito in un contesto sociale e culturale, con bisogni, aspirazioni, relazioni umane e affettive (3). In altre parole, emerge la necessità di valutare I'impatto che la malattia e le cure hanno sulla "qualità di vita" del paziente. Non è sufficiente raggiungere, come obiettivo, solo l'allungamento della vita, ossia quanto tempo il paziente sopravviverà alla sua malattia, ma è fondamentale preoccuparsi anche di come sarà la sua vita.

L'OMS, nel 1948, dà questa definizione di Qualità di Vita: "Qualità di vita è la percezione soggettiva che un individuo ha della propria posizione nella vita, nel contesto di una cultura e di un insieme di valori nei quali egli vive, anche in relazione ai propri obiettivi, aspettative e preoccupazioni». Riguarda quindi 
un concetto ad ampio spettro, che è modificabile in maniera complessa dalla percezione della propria salute fisica e psicologico-emotiva, dal livello di indipendenza, dalle relazioni sociali e dalla interazione con il proprio specifico contesto ambientale (4). Per gli usi medici, è più preciso e corretto parlare di "Qualità di Vita Correlata alla Salute" (Health Related Quality of Life, HQRL), definita come "l'insieme degli aspetti qualitativi della vita dell'individuo correlabili ai domini della malattia e della salute, e pertanto modificabili dalla medicina» (5).

\section{Background}

Gli strumenti generici per la valutazione della Qualità di Vita Correlata alla Salute ne misurano tutti gli aspetti generali. I vantaggi sono rappresentati dalla versatilità d'uso: possono essere applicati a popolazioni con diverse caratteristiche e a diverse patologie e permettono di confrontare diverse realtà all'interno di un programma sanitario. Lo svantaggio è che non sono adatti per essere applicati a realtà più specifiche (specifica popolazione, specifica patologia) (6). Tra gli strumenti generici il più noto è l'SF-36 Health Survey $(7,8)$. Lo strumento specifico per misurare la QdV nei pazienti affetti da insufficienza renale cronica in trattamento dialitico sostitutivo più moderno e attualmente disponibile è il Kidney Disease Quality of Life Short Form Instrument (KDQOL-SF) (9). Questo strumento è ben noto e ha contribuito in modo significativo allo sviluppo della comprensione della qualità di vita nella popolazione affetta da insufficienza renale cronica (10).

Nell'Azienda USL di Rimini sono attivi tre Centri di emodialisi: un Centro Ospedaliero (presso l'Ospedale Infermi di Rimini) e due Centri di Assistenza Limitata (presso l'Ospedale Ceccarini di Riccione e l'Ospedale Franchini di Santarcangelo di Romagna). È attivo inoltre un ambulatorio di dialisi peritoneale aperto 5 ore al giorno per 5 giorni a settimana.

II 31 dicembre 2013 a Rimini i pazienti in trattamento emodialitico cronico erano 193, quelli in dialisi peritoneale 13.

Presso l'Azienda USL di Rimini non è mai stata indagata la qualità della vita del paziente in dialisi: questo dato potrebbe fornire importanti informazioni all'Unità Operativa, utili per migliorare, potenziare, modificare ambiti del servizio offerto alla popolazione affetta da insufficienza renale cronica terminale.

\section{Materiali e Metodi}

\section{Obiettivi}

- Realizzare una valutazione aggiornata dello stato di salute e della QdV dei nostri pazienti dializzati quanto più obiettiva possibile.

- Individuare ambiti di salute qualitativamente migliorabili per il paziente in dialisi peritoneale, stabilendo un punto di partenza per il confronto dei dati nel futuro.

\section{Popolazione}

Sono stati selezionati 11 pazienti in trattamento dialitico peritoneale su un totale di 12 pazienti: uno è stato escluso in quanto giudicato non in grado di compilare il questionario autonomamente.

Sono stati successivamente individuati 11 pazienti in trattamento emodialitico cronico che rappresentassero il più possibile un campione simile secondo i criteri seguenti: genere, età media, grado di autonomia, rientro da trapianto, in lista per trapianto.

In entrambi i campioni, degli 11 pazienti totali 8 erano maschi e 3 erano femmine.

L'età media dei pazienti in dialisi peritoneale era di 59,63 anni; l'età media dei pazienti in dialisi extracorporea era di 65,7 anni: non è stato possibile infatti parificare questo dato tra i due gruppi.

Per quanto riguarda il grado di autonomia, 10 pazienti in dialisi peritoneale risultavano completamente autonomi, soltanto un paziente presentava un grado di autonomia incompleto; tutti gli 11 pazienti dell'emodialisi, invece, erano completamente autonomi.

In entrambi i campioni, risultava esserci un solo paziente in rientro da trapianto.

I pazienti in lista per trapianto in dialisi peritoneale erano 4, quelli in emodialisi 3.

\section{Strumenti}

Per valutare la qualità della vita dei pazienti dializzati nell'U.O. Nefrologia e Dialisi dell'Azienda USL di Rimini è stato organizzato un gruppo di studio e ricerca, formato da una studentessa infermiera, un Medico Nefrologo, un'Infermiera referente dell'Ambulatorio di Dialisi Peritoneale dell'Ospedale Infermi di Rimini e la Coordinatrice Infermieristica dell'U.O. Nefrologia e Dialisi dell'Ospedale Infermi di Rimini.

L'indagine è stata preceduta da una fase preparatoria per individuare il questionario di rilevazione della QdV più adatto alle nostre esigenze. II gruppo si è valso, quindi, del confronto con la letteratura, effettuando una ricerca bibliografica sulla dialisi peritoneale e qualità della vita su PubMed, utilizzando questi termini di ricerca: "peritoneal dialysis and health related quality of life not transplantation". Come limiti sono stati impostati: le "pubblicazioni degli ultimi 5 anni" e il "genere umano".

Sono stati trovati 41 articoli di cui 24 esclusi per varie ragioni (Tab. I), 17 quelli giudicati pertinenti. Di questi, 2 sono studi multicentrici, tutti sono indagini osservazionali.

In questi lavori è stata indagata la qualità della vita dei pazienti in dialisi basandosi in 9 indagini su due soli strumenti: il questionario Kidney Disease Quality of Life - Short Form version 1.3 (11-13) e lo Short Form Medical Outcomes Study SF36 o SF-12 (12, 14-18). Negli altri 8 studi lo strumento scelto per l'indagine è stato preparato dall'équipe che ha condotto l'indagine. 
TABELLA I - Risultati della ricerca bibliografica

Articoli trovati totali

41

Articoli esclusi perché riguardanti l'età pediatrica

3

Articoli esclusi perché non pertinenti

Articoli pertinenti

Nella letteratura consultata la qualità della vita correlata allo stato di salute in dialisi peritoneale viene percepita in maniera differente a seconda dell'età, in quanto la gerarchia dei bisogni si modifica in relazione ad essa; laddove veniva posto un confronto con una uguale popolazione di pazienti in emodialisi non c'erano sostanziali differenze nella percezione dei pazienti intervistati.

\section{Protocollo utilizzato}

In considerazione dei dati della letteratura, abbiamo deciso di creare un questionario di reparto servendoci del Kidney Disease Quality of Life - Short Form versione italiana 1.3 , lo strumento più specifico e attualmente più utilizzato per misurare la QdV dei pazienti uremici dializzati e delI'SF-12 Health Survey versione italiana. Abbiamo costruito, dunque, un questionario di 10 domande, di cui 4 estrapolate dall'SF-12 versione italiana e 5 appartenenti al KDQOL$S F$ versione italiana 1.3. Abbiamo scelto di aggiungere una domanda sul dolore (domanda 5), che non è presente né

TABELLA II - Aree indagate nel questionario

\begin{tabular}{lc}
\hline Aree indagate & $\begin{array}{c}\text { Numero di domande } \\
\text { per area }\end{array}$ \\
\hline Stato generale di salute & 1 \\
Limitazioni nelle attività & 3 \\
Stato emotivo & 1 \\
Interferenza nelle attività sociali & 1 \\
Dolore & 12 \\
Sintomi/problemi della malattia renale \\
cronica
\end{tabular}

nell'SF-12 né all'interno del KDQOL-SF, relativa al grado di dolore provato dal paziente nelle ultime 4 settimane.

II questionario definitivo è costituito, pertanto, da 31 items, i quali indagano gli ambiti riportati nella Tabella II.

Per quanto riguarda le risposte, sono state organizzate "a scala" partendo da quella più positiva (immediatamente a sinistra) alla più negativa (tutta spostata a destra), in modo da avere così un primo impatto visivo circa le concentrazioni delle risposte, permettendone anche un confronto tra i due campioni di popolazione.

Dopo aver redatto il questionario definitivo, si è proceduto alla sua distribuzione nel periodo dal 24 marzo al 2 maggio 2014; ogni paziente è stato informato riguardo la finalità del questionario, riguardo l'anonimato della raccolta dati e a ognuno è stato chiesto il consenso. I pazienti in trattamento dialitico peritoneale, che si presentavano all'ambulatorio per la visita mensile o gli esami ematici, sono stati accompagnati in una saletta dedicata, non solo per garantire l'anonimato e la privacy e per evitare distrazioni ma, soprattutto, per non essere influenzati dagli operatori sanitari presenti. Al termine della compilazione il questionario veniva inserito dal paziente stesso direttamente in una scatola di raccolta. Ai pazienti in emodialisi, invece, il questionario è stato distribuito durante il trattamento dialitico, ma gli è stato spiegato di compilarlo a casa; questo per non invadere il loro spazio e per evitare che si confrontassero fra di loro, influenzandosi nelle risposte. Al momento della restituzione, il paziente ha inserito il questionario nella scatola di raccolta.

\section{Limiti dello studio}

Il questionario composto nella maniera sopra indicata è originato da modifiche apportate a strumenti validati; modifiche necessarie per rispondere ad esigenze interne di approfondimento delle conoscenze relative ad ambiti specifici quali il dolore e la qualità del servizio percepita dall'utenza. Non è stato sottoposto a opportuna validazione ufficiale.

\section{Risultati}

Sono stati distribuiti 22 questionari, di cui 11 in dialisi peritoneale e 11 in emodialisi; sono stati raccolti dalla scatola dell'ambulatorio peritoneale tutti gli 11 questionari distribuiti; anche da quella dell'emodialisi ne sono stati raccolti 11 , per un totale percentuale del $100 \%$.

Di seguito sono riportate le differenti risposte suddivise nei due gruppi. 
1 - In generale direbbe che la sua salute è:

\begin{tabular}{|l|c|c|c|c|c|c|}
\hline & $\begin{array}{c}\mathbf{1} \\
\text { Eccellente }\end{array}$ & $\begin{array}{c}\mathbf{2} \\
\text { Molto buona }\end{array}$ & $\begin{array}{c}\mathbf{3} \\
\text { Buona }\end{array}$ & $\begin{array}{c}\mathbf{4} \\
\text { Passabile }\end{array}$ & $\begin{array}{c}\mathbf{5} \\
\text { Scadente }\end{array}$ & $\begin{array}{c}\text { Non risponde } \\
\text { Emodialisi }\end{array}$ \\
\hline Dialisi Peritoneale & & 2 & 3 & 5 & 2 & 2 \\
\hline
\end{tabular}

2 - Il suo stato di salute attuale limita le attività di moderato impegno fisico, come salire le scale, spostare un tavolo, usare l'aspirapolvere, fare un giretto in bicicletta, giocare a bocce?

\begin{tabular}{|l|c|c|c|c|c|c|}
\hline & $\begin{array}{c}\mathbf{1} \\
\text { Per niente }\end{array}$ & $\begin{array}{c}\mathbf{2} \\
\text { Molto poco }\end{array}$ & $\begin{array}{c}\mathbf{3} \\
\text { Abbastanza }\end{array}$ & $\begin{array}{c}\mathbf{4} \\
\text { Molto }\end{array}$ & $\begin{array}{c}\mathbf{5} \\
\text { Moltissimo }\end{array}$ & Non risponde \\
\hline Emodialisi & & 4 & 4 & & 3 & \\
\hline Dialisi Peritoneale & 2 & 4 & 4 & 1 & & \\
\hline
\end{tabular}

3 - Per quanto tempo nelle ultime settimane si è sentito...

3a - Calmo e sereno

\begin{tabular}{|l|c|c|c|c|c|c|}
\hline & $\begin{array}{c}\mathbf{1} \\
\text { Sempre }\end{array}$ & $\begin{array}{c}\mathbf{2} \\
\text { Quasi sempre }\end{array}$ & $\begin{array}{c}\mathbf{3} \\
\text { Una parte del } \\
\text { tempo }\end{array}$ & $\begin{array}{c}\mathbf{4} \\
\text { Quasi mai }\end{array}$ & $\begin{array}{c}\mathbf{5} \\
\text { Mai }\end{array}$ & $\begin{array}{c}\text { Non risponde } \\
\text { Emodialisi }\end{array}$ \\
\hline Dialisi Peritoneale & 1 & 4 & 4 & 3 & \\
\hline
\end{tabular}

3b - Pieno di energia

\begin{tabular}{|l|c|c|c|c|c|c|}
\hline & $\begin{array}{c}\mathbf{1} \\
\text { Sempre }\end{array}$ & $\begin{array}{c}\mathbf{2} \\
\text { Quasi sempre }\end{array}$ & $\begin{array}{c}\mathbf{3} \\
\text { Una parte del } \\
\text { tempo }\end{array}$ & $\begin{array}{c}\mathbf{4} \\
\text { Quasi mai }\end{array}$ & $\begin{array}{c}\mathbf{5} \\
\text { Mai }\end{array}$ & $\begin{array}{c}\text { Non risponde } \\
\text { Emodialisi }\end{array}$ \\
\hline Dialisi Peritoneale & 1 & 2 & 3 & 3 & 2 & \\
\hline
\end{tabular}

3c - Scoraggiato e triste

\begin{tabular}{|l|c|c|c|c|c|c|}
\hline & $\begin{array}{c}\mathbf{1} \\
\text { Mai }\end{array}$ & $\begin{array}{c}\mathbf{2} \\
\text { Quasi mai }\end{array}$ & $\begin{array}{c}\mathbf{3} \\
\text { Una parte del } \\
\text { tempo }\end{array}$ & $\begin{array}{c}\mathbf{4} \\
\text { Quasi sempre }\end{array}$ & $\begin{array}{c}\mathbf{5} \\
\text { Sempre }\end{array}$ \\
\hline Emodialisi & 1 & 3 & 6 & & 1 & \\
\hline Dialisi Peritoneale & 1 & 4 & 4 & 2 & & \\
\hline
\end{tabular}

4 - Nelle ultime 4 settimane per quanto tempo la sua salute fisica o il suo stato emotivo hanno interferito nelle sue attività sociali, in famiglia o con gli amici?

\begin{tabular}{|l|c|c|c|c|c|}
\hline & $\begin{array}{c}\mathbf{1} \\
\text { Mai }\end{array}$ & $\begin{array}{c}\mathbf{2} \\
\text { Quasi mai }\end{array}$ & $\begin{array}{c}\mathbf{3} \\
\text { Una parte del } \\
\text { tempo }\end{array}$ & $\begin{array}{c}\mathbf{4} \\
\text { Quasi sempre }\end{array}$ & $\begin{array}{c}\mathbf{5} \\
\text { Sempre }\end{array}$ \\
\hline Emodialisi & 1 & 4 & 2 & 4 & \\
\hline Dialisi Peritoneale & 1 & 5 & 5 & & \\
\hline
\end{tabular}

5 - Nelle ultime 4 settimane ha provato dolore? Ne descriva l'intensità usando la scala proposta qui sotto:

\begin{tabular}{|l|l|l|l|l|l|l|l|l|l|l|l|l|}
\hline & $\mathbf{0}$ & $\mathbf{1}$ & $\mathbf{2}$ & $\mathbf{3}$ & $\mathbf{4}$ & $\mathbf{5}$ & $\mathbf{6}$ & $\mathbf{7}$ & $\mathbf{8}$ & $\mathbf{9}$ & $\mathbf{1 0}$ & Non risponde \\
\hline Emodialisi & 1 & & & 2 & & 3 & 2 & 1 & & & 2 \\
\hline $\begin{array}{l}\text { Dialisi } \\
\text { Peritoneale }\end{array}$ & 2 & & 2 & 4 & & 2 & & & & 1 & \\
\hline
\end{tabular}


6 - Queste domande si riferiscono a come si è sentito e a come le sono andate le cose durante le ultime $\mathbf{4}$ settimane. Risponda a ogni domanda nella maniera che più si avvicina a come si è sentito.

Nel corso delle ultime $\mathbf{4}$ settimane quanto è stato disturbato da:

\begin{tabular}{|c|c|c|c|c|c|c|}
\hline & $\begin{array}{l}\text { Per nulla } \\
\text { disturbato }\end{array}$ & $\begin{array}{c}\text { Un po' } \\
\text { disturbato }\end{array}$ & $\begin{array}{l}\text { Abbastanza } \\
\text { disturbato }\end{array}$ & $\begin{array}{l}\text { Parecchio } \\
\text { disturbato }\end{array}$ & $\begin{array}{l}\text { Moltissimo } \\
\text { disturbato }\end{array}$ & Non risponde \\
\hline \multicolumn{7}{|c|}{ Dolenzia muscolare? } \\
\hline Emodialisi & 3 & 4 & 1 & 2 & 1 & \\
\hline Dialisi Peritoneale & 1 & 5 & 3 & 1 & & 1 \\
\hline \multicolumn{7}{|l|}{ Dolore al petto? } \\
\hline Emodialisi & 9 & 1 & & & 1 & \\
\hline Dialisi Peritoneale & 7 & 3 & & & & 1 \\
\hline \multicolumn{7}{|l|}{ Crampi? } \\
\hline Emodialisi & 3 & 5 & 1 & 1 & 1 & \\
\hline Dialisi Peritoneale & 5 & 4 & 2 & & & \\
\hline \multicolumn{7}{|l|}{ Prurito? } \\
\hline Emodialisi & & 5 & 3 & 3 & & \\
\hline Dialisi Peritoneale & 3 & 6 & 2 & & & \\
\hline \multicolumn{7}{|l|}{ Pelle secca? } \\
\hline Emodialisi & 1 & 2 & 4 & 3 & 1 & \\
\hline Dialisi Peritoneale & 2 & 3 & 5 & 1 & & \\
\hline \multicolumn{7}{|l|}{ Mancanza di fiato? } \\
\hline Emodialisi & 5 & 4 & 1 & 1 & & \\
\hline Dialisi Peritoneale & 3 & 6 & 1 & 1 & & \\
\hline \multicolumn{7}{|c|}{ Debolezza o capogiro? } \\
\hline Emodialisi & 5 & 2 & 2 & & 2 & \\
\hline Dialisi Peritoneale & 4 & 3 & 2 & 2 & & \\
\hline \multicolumn{7}{|c|}{ Mancanza di appetito? } \\
\hline Emodialisi & 7 & 2 & & 1 & 1 & \\
\hline Dialisi Peritoneale & 6 & 4 & 1 & & & \\
\hline \multicolumn{7}{|l|}{ Esausto o sfinito? } \\
\hline Emodialisi & 3 & 5 & & 1 & 2 & \\
\hline Dialisi Peritoneale & 3 & 4 & 2 & 2 & & \\
\hline \multicolumn{7}{|c|}{ Mani o piedi i intorpiditi? } \\
\hline Emodialisi & 4 & 2 & 2 & 1 & 2 & \\
\hline Dialisi Peritoneale & 2 & 6 & 1 & 2 & & \\
\hline \multicolumn{7}{|c|}{ Nausea, stomaco sottosopra? } \\
\hline Emodialisi & 5 & 3 & 2 & & 1 & \\
\hline Dialisi Peritoneale & 6 & 2 & 2 & 1 & & \\
\hline \multicolumn{7}{|c|}{$\begin{array}{l}\text { (Solo per pazienti in Emodialisi): } \\
\text { Problemi con la fistola? }\end{array}$} \\
\hline Emodialisi & 5 & 3 & & 1 & 2 & \\
\hline \multicolumn{7}{|c|}{$\begin{array}{l}\text { (Solo per pazienti in Dialisi Perito- } \\
\text { neale): Problemi conil catetere? }\end{array}$} \\
\hline Dialisi Peritoneale & 9 & 2 & & & & \\
\hline
\end{tabular}


7 - Alcune persone sono disturbate nella loro vita quotidiana dalle conseguenze della malattia renale e altre no. Quanto è disturbato dalla sua malattia renale per ognuno di questi aspetti?

\begin{tabular}{|c|c|c|c|c|c|c|}
\hline & $\begin{array}{l}\text { Per nulla } \\
\text { disturbato }\end{array}$ & $\begin{array}{l}\text { Un po' } \\
\text { disturbato }\end{array}$ & $\begin{array}{l}\text { Abbastanza } \\
\text { disturbato }\end{array}$ & Parecchio disturbato & $\begin{array}{l}\text { Moltissimo } \\
\text { disturbato }\end{array}$ & $\begin{array}{l}\text { Non } \\
\text { risponde }\end{array}$ \\
\hline \multicolumn{7}{|l|}{ Ridurre i liquidi? } \\
\hline Emodialisi & 4 & & 3 & 2 & 2 & \\
\hline Dialisi Peritoneale & 4 & 4 & 3 & & & \\
\hline \multicolumn{7}{|l|}{ Stare a dieta? } \\
\hline Emodialisi & 3 & 4 & 1 & 2 & 1 & \\
\hline Dialisi Peritoneale & 4 & 2 & 4 & 1 & & \\
\hline \multicolumn{7}{|l|}{ Attività in casa? } \\
\hline Emodialisi & 3 & 2 & 1 & 3 & 2 & \\
\hline Dialisi Peritoneale & 3 & 5 & 1 & 1 & & 1 \\
\hline \multicolumn{7}{|l|}{ Viaggiare? } \\
\hline Emodialisi & 3 & 4 & 1 & 2 & 1 & \\
\hline Dialisi Peritoneale & & 1 & 1 & 4 & 2 & 3 \\
\hline \multicolumn{7}{|c|}{$\begin{array}{l}\text { Dipendere dai medici o da altro } \\
\text { personale sanitario? }\end{array}$} \\
\hline Emodialisi & 4 & 5 & & 1 & 1 & \\
\hline Dialisi Peritoneale & 7 & 1 & & 3 & & \\
\hline \multicolumn{7}{|c|}{$\begin{array}{l}\text { Stress e preoccupazioni legate } \\
\text { alla malattia renale? }\end{array}$} \\
\hline Emodialisi & 2 & 3 & 2 & 2 & 2 & \\
\hline Dialisi Peritoneale & 1 & 6 & 2 & 2 & & \\
\hline \multicolumn{7}{|l|}{ Vita sessuale? } \\
\hline Emodialisi & 4 & 3 & & 1 & 1 & 2 \\
\hline Dialisi Peritoneale & 1 & 3 & 3 & 1 & 1 & 2 \\
\hline \multicolumn{7}{|l|}{ Aspetto fisico? } \\
\hline Emodialisi & 7 & 2 & 1 & & 1 & \\
\hline Dialisi Peritoneale & 2 & 5 & 1 & 1 & 1 & 1 \\
\hline
\end{tabular}

8 - Con la domanda successiva le chiediamo di valutare come dorme, utilizzando una scala che va da 0, che rappresenta un sonno "Molto buono", a 10, che rappresenta un sonno "Pessimo".

Per esempio, se ritiene che la qualità del suo dormire sia a metà strada fra "Pessimo" e "Molto buono", faccia una crocetta nella casella sotto al numero 5. Se ritiene che il suo sonno sia un po' meglio del valore 5, segni 4. Se ritiene che il suo sonno sia un po' peggio del livello 5, segni 6 (e così via).

Come valuterebbe in generale il suo sonno, su una scala da 0 a 10 ?

[faccia una x nella casella]

\begin{tabular}{|c|c|c|c|c|c|c|c|c|c|c|c|c|}
\hline & 0 & 1 & 2 & 3 & 4 & 5 & 6 & 7 & 8 & 9 & 10 & $\begin{array}{c}\text { Non } \\
\text { risponde }\end{array}$ \\
\hline Emodialisi & & 2 & & 1 & & 3 & & 2 & & 2 & 1 & \\
\hline $\begin{array}{l}\text { Dialisi } \\
\text { Peritoneale }\end{array}$ & 1 & 1 & & 1 & 2 & 4 & 1 & 1 & & & & \\
\hline
\end{tabular}


9 - Prendiamo in considerazione le cure che riceve per la dialisi. Dal punto di vista della sua personale soddisfazione, come valuta disponibilità e interesse mostrati nei suoi confronti?

\begin{tabular}{|l|c|c|c|c|c|c|c|}
\hline & Ottima & Molto buona & Buona & Discreta & Passabile & Scarsa & Non risponde \\
\hline Emodialisi & 6 & 1 & 2 & 1 & & 1 \\
\hline $\begin{array}{l}\text { Dialisi } \\
\text { Peritoneale }\end{array}$ & 7 & 1 & 3 & & & \\
\hline
\end{tabular}

10 - Quanto vere o false sono queste affermazioni?

\begin{tabular}{|c|c|c|c|c|c|c|}
\hline & $\begin{array}{l}\text { Assolutamente } \\
\text { vera }\end{array}$ & $\begin{array}{l}\text { In genere } \\
\text { vera }\end{array}$ & Non so & $\begin{array}{l}\text { In genere } \\
\text { falsa }\end{array}$ & $\begin{array}{l}\text { Assolutamente } \\
\text { falsa }\end{array}$ & $\begin{array}{l}\text { Non } \\
\text { risponde }\end{array}$ \\
\hline \multicolumn{7}{|c|}{$\begin{array}{l}\text { Il personale della dialisi mi incoraggia } \\
\text { ad essere autonomo }\end{array}$} \\
\hline Emodialisi & 5 & 4 & 2 & & & \\
\hline Dialisi Peritoneale & 10 & 1 & & & & \\
\hline \multicolumn{7}{|c|}{$\begin{array}{l}\text { Il personale della dialisi mi aiuta ad } \\
\text { affrontare la mia malattia renale }\end{array}$} \\
\hline Emodialisi & 6 & 4 & 1 & & & \\
\hline Dialisi Peritoneale & 10 & 1 & & & & \\
\hline
\end{tabular}

\section{Discussione}

Sebbene le ricerche effettuate in letteratura ci abbiano mostrato risultati non uniformi, si può affermare che all'interno dell'Azienda USL di Rimini il campione di pazienti in trattamento dialitico peritoneale dimostra di avere una Qualità di Vita migliore rispetto al campione di pazienti emodializzati e questo su quasi tutti i fronti. Infatti relativamente al dolore percepito, alla qualità del sonno e alla presenza di sintomi disturbanti i pazienti in emodialisi presentano una piccola percentuale di risposte che si attesta sui valori "Molto negativi" posizionati più a destra nella Tabella delle risposte. Un discorso a parte va riservato alla vita sessuale e al viaggiare. Mentre questi aspetti non sembrano infatti essere intaccati dall'insufficienza renale nei pazienti in trattamento emodialitico, per la popolazione in trattamento peritoneale costituiscono un importante problema, e sono tra gli unici items, insieme all'aspetto fisico, per cui i pazienti hanno riempito questa volta anche il campo prettamente negativo ("Moltissimo disturbato").

Quasi il 60\% delle risposte relative al viaggiare sono concentrate nei campi "Parecchio disturbato" - "Moltissimo disturbato", tenendo conto poi che 3 pazienti non hanno risposto alla domanda. Questo dato non può e non dev'essere trascurato. La dialisi peritoneale consente a tutti i pazienti di viaggiare, ovviamente questo comporta il trasporto del materiale necessario per effettuare il trattamento. I risultati dei questionari ci portano a fare delle ipotesi. Prima di tutto, può darsi che i pazienti sottoposti all'indagine non abbiano ben compreso o siano addirittura ignari di questo particolare vantaggio della dialisi peritoneale (perché magari tra le tante informazioni date, questa è loro sfuggita), ossia pensano di essere limitati nell'organizzare le loro vacanze a causa del trattamento, in realtà sono piuttosto liberi sotto quest'aspetto, potendo andare pressoché ovunque a patto di portare il materiale con sé. Se cosi fosse, occorrerebbe fornire al paziente tutte le informazioni necessarie al fine di renderlo consapevole che la dialisi peritoneale, in quanto trattamento domiciliare, lo rende piuttosto libero, anche di viaggiare. Un'altra ipotesi è che il problema sia costituito proprio dal trasporto del materiale, cioè il paziente sa di poter effettuare viaggi, ma il fatto di doversi portare dietro tutti gli strumenti costituisce per lui un ostacolo importante. In questo caso, l'infermiere dovrebbe incoraggiare il paziente, facendogli capire che tutte quelle cose da lui considerate indaginose servono in realtà per farlo stare bene e fanno parte di lui, ma non sono e non devono costituire un limite all'organizzare viaggi che il paziente deve continuare a fare, soprattutto se da lui tanto graditi.

Per quel che riguarda la vita sessuale, invece, la maggioranza delle risposte fornite dai pazienti in trattamento peritoneale (più del 50\%), a differenza delle risposte date dagli emodializzati, si concentra nei campi "Un po' disturbato" "Abbastanza disturbato". Tuttavia tutto ciò è comprensibile, poiché nel campione sono presenti anche persone giovani, ed è pertanto inevitabile che la presenza di un catetere in addome possa recare un minimo di disturbo sotto questo aspetto.

\section{Conclusioni}

Lo studio effettuato non ha la pretesa di affermare la superiorità di una tecnica dialitica rispetto all'altra, anche perché 
spesso, più della preferenza del singolo paziente, sono le sue caratteristiche fisiche e psico-socio-attitudinali a suggerire quale possa essere la metodica più adatta a lui e alle sue esigenze. II questionario sarà eventualmente validato in un prossimo studio. L'auspicio di questo lavoro, invece, è che esso possa essere il punto di partenza di un percorso che metta al centro il paziente uremico e la sua qualità di vita in un contesto in cui quest'ultima ne risente inevitabilmente. Laddove, come documenta la nostra indagine, esista una metodica come la dialisi peritoneale di pari efficacia all'emodialisi ma che dimostri di avere meno "ripercussioni" sulla vita del soggetto, questa deve essere assolutamente presa in considerazione dallo staff e deve essere proposta ai pazienti.

\section{Disclosures}

Financial support: No financial support was received for this submission.

Conflict of interest: The authors have no conflict of interest.

\section{Bibliografia}

1. Limido A, Nichelatti M, Postorino M, et al. Come cambia la popolazione uremica nel Registro Nazionale di Dialisi e Trapianto. G Ital Nefrol 2012;29(S58):S22.

2. Santoro A. Registro Regionale Emiliano-Romagnolo di Dialisi e Trapianto (RER-DT) - Report 2010. Servizio Sanitario Regionale Emilia-Romagna. 2012;6-7.

3. De Franchis R. Cure mediche e qualità della vita: una nuova frontiera. Argomento trattato nell'assemblea straordinaria dei soci di A.M.I.C.I., Lombardia, 20 novembre 1999.

4. Fiore $\mathrm{C}$. Un progetto di ricerca sulla qualità della vita in oncologia. Considerazioni generali ed iniziali osservazioni personali. Prendersi cura, vol. 1, 2009:2.

5. Istituto Superiore di Sanità, Dipartimento del Farmaco. Qualità della vita e farmacoeconomia: outcomes necessari per una migliore valutazione di strategie terapeutiche in ambito sperimentale e nella pratica clinica;p.7.

6. Istituto Superiore di Sanità, Dipartimento del Farmaco. Quali- tà della vita e farmacoeconomia: outcomes necessari per una migliore valutazione di strategie terapeutiche in ambito sperimentale e nella pratica clinica;p. 15.

7. Ware JE, Sherbourne CD. The MOS 36 item short-form health survey (SF-36). I. Conceptual framework and item selection. Med Care. 1992;30(6):473-83.

8. Brazier JE, Harper R, Jones NM, et al. Validating the SF-36 health survey questionnaire: new outcome measure for primary care. BMJ. 1992;305(6846):160-4.

9. Hays RD, et al. Kidney Disease Quality of Life Short Form (KQDOL-SF ${ }^{\mathrm{TM}}$ ), Version 1.3: A Manual for Use and Scoring. Santa Monica (CA): RAND Corporation 1997; p.1.

10. Hyde C, Tobita I. La ricerca sulla qualità della vita: uno strumento prezioso per l'infermiere nefrologico. Journal of Renal Care 2007;XXXIII1:28.

11. Okpechi IG, Nthite T, Swanepoel CR. Health-related quality of life in patients on hemodialysis and peritoneal dialysis. Saudi J Kidney Dis Transpl. 2013;24(3):519-26.

12. Abreu MM, Walker DR, Sesso RC, Ferraz MB. Health-related quality of life of patients recieving hemodialysis and peritoneal dialysis in São Paulo, Brazil: a longitudinal study. Value Health. 2011;14(5 Suppl 1):S119-21.

13. Varela L, Vázquez MI, Bolaños L, Alonso R. Psychological predictors for health-related quality of life in patients on peritoneal dialysis. Nefrologia. 2011;31(1):97-106.

14. Kim JY, Kim B, Park KS, et al. Health-related quality of life with KDQOL-36 and its association with self-efficacy and treatment satisfaction in Korean dialysis patients. Qual Life Res. 2013; 22(4):753-8.

15. Park HC, Lee $\mathrm{H}$, Lee JP, et al. Lower residual renal function is a risk factor for depression and impaired health-related quality of life in Korean peritoneal dialysis patients. J Korean Med Sci. 2012;27(1):64-71.

16. Turkmen K, Yazici R, Solak Y, et al. Health-related qualıty of Iıfe, sleep qualıty, and depression in peritoneal dialysis and hemodialysis patients. Hemodial Int. 2012;16(2):198-206.

17. Meinero S, Tesio E, Bainotti S, et al. [Quality of life assessment in dialyzed patients in the Cuneo area]. G Ital Nefrol. 2011;28(1):72-9. Italian.

18. Russo GE, Morgia A, Cavallini M, et al. [Quality of life assessment in patients on hemodialysis and peritoneal dialysis]. G Ital Nefrol. 2010;27(3):290-5. Italian. 